\title{
PESQUISA-AÇÃO: contribuição para prática investigativa do enfermeiro ${ }^{a}$
}

\author{
Claudete Ferreira de Sousa M ONT EIRO ${ }^{b}$, M aria Rosilene Cândido M OREIRAc, \\ E dina A raújo Rodrigues OL IVE IRA d, M aria E dileuza Soares M OU RA ${ }^{e}$, J onhatan Vel oso COSTA
}

\section{RESUMO}

E ste artigo apresenta uma reflexão teórica sobre a metodologia da pesquisa-ação enquanto estratégia alternativa ao modelo convencional de investigação científica. Contextualiza a metodologia a partir de dois estudiosos da temática, que propõem modelos estruturais distintos: o ciclo de investigação-ação e o modelo de fases flexíveis. A ponta exemplos de utilização da pesquisa-ação a partir de estudos realizados por enfermeiros. Considera-se modalidade que vem sendo cada vez mais adotada nos espaços do cuidado, bem como no processo educativo, apontando para a necessidade de maior aprofundamento teórico, a fim de que a estratégia possa ser ferramenta útil na prática investigativa do enfer meiro.

D escritores: M etodologia. Pesquisa qualitativa. Pesquisa em enfermagem.

\section{RESUMEN}

E I artículo presenta una reflexión teórica acerca de la metodología de la investigación - acción sobre la estrategia alter nativa del modelo convencional dela investigación científica. Contextualiza la metodología a partir de dos especialistas dela temática, que proponen model os estructurales distintos: el ciclo de investigación-acción y el modelo de fases flexibles. A punta ejemplos dela utilización de la investigaciónacción a partir de estudios realizados por enfermer os en áreas de desar rollo profesional. Se considera una modalidad que viene si endo cada vez más adoptada en las áreas del cuidado, así como en el proceso educativo, apuntándo a la necesidad de mayor profundización teórica, a fin de quela estrategia pueda ser una her ramienta útil en la práctica investigativa del enfermero.

Descriptores: M etodología. I nvestigación cualitativa. Investigación en enfermería.

T ítulo: Investigación-acción: contribución en la práctica investigativa del enfermero.

\section{ABST RACT}

T he present paper shows a theor etical reflection on action research methodology as an alter native strategy to the conventional model of scientific investigation. M ethodology is discussed from the per spective of tw 0 schol ars who propose tw o different str uctural models: actioninvestigation cycle and flexiblestage model. We provide examples of the use of action research from studies car ried out by nurses. Action research is an emergent model which is being more and more adopted in educational and nursing fields, reflecting the necessity of deeper theoretical knowledge and revealing itself as a useful instrument in nursing research.

D escriptors: M ethodology. Qualitative research. N ursing research.

T itle: R esearch action: a contribution to nurses' investigative practices.

\footnotetext{
a M etodologia de pesquisa utilizada pelas autoras no Projeto "Intervenções terapêuticas em saúde mental: uma proposta para redução do estigma", desenvolvido na U niversidade Federal do Piauí (U F PI), Campus Senador Helvídio N unes de Barros.

${ }^{b}$ D outora em Enfermagem, Professora Adjunta do Curso de G raduação e do Programa de Pós-G raduação M estrado em Enfermagem da UFPI, Teresina, Piauí, Brasil.

'E nfermeira E specialista em Saúde da Família e em Saúde M ental, M estranda em Saúde Coletiva pela U niver sidade Federal de São Paulo (U N IFESP), Professora Auxiliar do Curso de Enfermagem da UFPI, Campus Senador Helvídio N unes de Barros, Bolsista do Projeto "Inter venções terapêuticas em saúde mental: uma proposta para redução do estigma" (CN Pq/ U F PI), Picos, Piauí, Brasil.

d E nfermeira E specialista em Saúde da Família, Professora A uxiliar do Curso de Enfermagem da U F PI, Campus Senador H elvídio N unes de Barros, Bolsista do Projeto "I ntervenções ter apêuticas em saúde mental: uma proposta para redução do estigma” (CN P q/ U F PI), Picos, Piauí, Brasil.

e E nfermeira, M estranda do Programa de Pós-Graduação M estrado em Enfermagem da U niversidade Federal do Piauí, T eresina, Piauí, Brasil.

f Graduando em Enfermagem da U FPI, Bolsista Iniciação Científica/ CN P q, Picos, Piauí, Brasil.
} 


\section{INT RODUÇÃO}

F ruto da consolidação da Enfer magem como saber científico a partir dos constructos deF lorence Nightingale, denominada Enfermagem M oderna, as práticas profissionais dos enfer meiros fundaramse em conceitos e teorias específicas da profissão, exigindo-se que esses profissionais adotassem postura crítica e reflexiva, utilizando o raciocínio crítico para a tomada de decisões ${ }^{(1)}$.

T al exigência fez despontar, no âmbito da comunidade de E nfermagem, pressupostos diversos, cuja contribuição significou a el abor ação do corpus teórico da profissão, favorecendo ao enfermeiro 0 fazer articulado ao pensar, sendo esse binômio ressignificado e ampliado constantemente pelo conhecimento produzido através da pesquisa ${ }^{(1)}$.

Sob este prisma, a produção do conhecimento em E nfermagem tem se apropriado do método científico como eixo estruturante, a partir do qual os problemas do cotidiano de trabalho são explorados, buscando-se solucioná-los. 0 processo investigativo ocorre, então, como elemento cada vez mais essencial, na práxis do enfermeiro.

Consider ada área integ rante das Ciências Sociais, a E nfermagem se apropria de alguns métodos de pesquisa social para investigar fenômenos pertinentes a prática profissional. D entre as abordagens utilizadas (qual itativa e quantitativa), a qualitativa tem sido amplamente adotada pelo fato de permitir ao enfermeiro vislumbrar a realidade, sem a interferência dos cálculos estatísticos. Consiste na investigação dos aspectos subjetivos, que incluem os significados, as crenças e as atitudes humanas $^{(2)}$, conferindo sua característica primordial de compreender o fenômeno a partir dos sujeitos nele envolvidos. Assim, a abordagem qualitativa per mite ao enfer meiro a descober ta dos campos empíricos fenomenológicos, a adoção de estratégias metodológicas específicas, utilização de técnicas e instrumentos de pesquisa, através dos quais é mantidos o rigor científico e os resultados da investigação podem ser evidenciados.

As pesquisas são também classificadas em virtude do objetivo ao qual se propõem em exploratórias, descritivas e explicativas. D entre as pesquisas realizadas pel os enfer meiros, destacam-se a do tipo exploratória, que tem como escopo aproximar o pesquisador do objeto de estudo, possibilitando maior familiaridade em relação ao fenômeno; e a do tipo descritiva, que visa descrever com exati- dão a realidade estudada a partir das variáveis estabelecidas, favorecendo a compreensão da mesma pelas car acterísticas apresentadas e sua relação com o problema proposto ${ }^{(3)}$. A l guns estudos são desenhados considerando-se simultaneamente as duas classificações, caracterizando o tipo exploratóriodescritivo, através do qual o pesquisador consegue tornar o fenômeno conhecido, estabelecer hipóteses e ainda perceber a dinâmica relacional existente entre as variáveis emergentes e o problema investigado.

$\mathrm{N}$ esse contexto, o método adotado, juntamente com o tipo de pesquisa escolhido e os procedimentos empregados para operacionalizar a coleta e a análise das infor mações per mite definir o delineamento do estudo. A ssim, tem-se a pesquisa bibliog ráfica, documental, ex- post-facto e participante como exemplos de delineamentos utilizados.

U ma estratégia alternativa de pesquisa, denominada pesquisa-ação, tem sido empregada nos estudos desenvolvidos no campo da saúde, bem como na Enfermagem, como uma proposta que se adequou ao método qualitativo, proporcionando uma interação entre o pesquisador e o sujeito investigado. $N$ esse método o conhecimento trazido pel o sujeito torna-se ponto de partida para a reflexão e reconstrução desse conhecimento por meio do processo participativo.

A pesquisa-ação é, pois um tipo de pesquisa aplicada no campo das Ciências Sociais significando um processo simultâneo de investigação e ação, cuja intenção primordial é o conhecimento e a resolução do problema coletivo a partir dos fatos observados, culminando na transformação dos pesquisadores e dos participantes envolvido no contexto social, sítio do problema ${ }^{(4)}$. É uma estratégia metodológica para as pesquisas aplicadas, pois se direciona para a identificação de problemas e respectivas soluções ${ }^{(5)}$, constituindo el emento favorável e útil nas investigações da área de Enfermagem.

E ste estudo se propõe a apresentar uma reflexão teórica sobre a metodologia da pesquisa-ação, destacando seu planejamento e elaboração, bem como sua aplicabilidade na prática investigativa do enfermeiro.

\section{PESQUISAS SOCIAIS: do método convencional para uma proposta alternativa}

No contexto da investigação científica, o método quantitativo tem sido tradicionalmente ado- 
tado em estudos da área de saúde, a fim de evidenciar sua importância na generalização dos fatos inferidos. Contudo, nas pesquisas qualitativas, diver sos temas têm sido explorados, conferindo igual relevância, por meio da riqueza de informações e da abrangência que esses estudos propiciam.

A Enfermagem busca ampliar o corpus teórico da profissão por meio da realização de pesquisas tanto quantitativas quanto qual itativas, viabilizadas por estratégias metodológicas que melhor se adequem ao tipo escolhido. D entre essas estratégias está a pesquisa-ação, que é utilizada quando há interesse coletivo na resolução de problemas $^{(6)}$.

A origem deste tipo de pesquisa ainda é incerta, no entanto atribui-se a autoria do termo a Kurt L ewin, psicólogo al emão que na década de 40 utilizava essa estratégia em seu trabal ho de treinamento de grupos de mães norte-americanas, denominados T-groups (grupos de treinamento) $)^{(3,7,8)}$. E ssas mulheres er am investigadas quanto aos seus hábitos e preferências alimentares no intuito de modificá-los ${ }^{(9)}$.

Cronologicamente, a trajetória dessa metodologia de pesquisa está ancorada em dois períodos, sendo o marco referencial do primeiro período as concepções de K urt L ewin (1940-1960), após o qual se inicia o segundo período, chegando aos dias atuais ${ }^{(10)}$.

U $m$ aspecto desta modalidade de pesquisa é que a investigação geral mente decorre da relação estabel ecida entre um grupo de pesquisadores eum grupo de sujeitos da investigação, através de momentos de vivência e discussão entre ambos, atingindo assim, dois tipos de objetivos: os práticos, por meio de levantamento de propostas de ação a fim de solucionar o problema posto; e os de conhecimento, proporcionando a ampliação do conhecimento de determinadas situações que seriam difícil acesso pela metodologia convencional (4).

Convém estabelecer al guns aspectos que meIhor exibem a diferenciação entre pesquisa convencional e pesquisa-ação. No primeiro tipo de pesquisa, são valorizados os aspectos individuais, a partir da coleta de opiniões, atitudes, motivações e comportamentos, que podem ser obtidos pela aplicação de questionários, entrevistas ou escalas de valores, não ocorrendo à busca de transformação da situação real. Porém, na pesquisa-ação, estudamse dinamicamente os problemas, decisões, conflitos e tomadas de consciência que se fazem durante o processo de transformação da realidade encontrada. N essa pesquisa, adotam-se o seminário, as reuniões e, quando pertinentes, a entrevista e a documentação, bem como as técnicas para diagnóstico situacional, resolução de problemas, mapeamento de representações e técnicas de divulgação e/ ou comunicação, confor me a final idade básica do estudo.

Evidencia-se, portanto, que a pesquisa-ação apropria-se de métodos e técnicas da pesquisa social, tornando-se uma estratégia complexa e abrangente, de caráter coletivo, participativo e ativo na obtenção de informações e na tomada de decisões para transfor mação da realidade.

A lguns autores utilizam o ter mo pesquisa participante e pesquisa-ação como expressões sinônimas, porém, percebe-se que há uma sutil diferença entre esses dois modos de investigação, requerendo maior aprofundamento nesses conceitos.

$\mathrm{N}$ a pesquisa-ação entende-se que, além da participação dos atores sociais, existe uma forma de ação planejada que pode assumir aspecto social, educacional, técnico ou outro, que nem sempre ocorre nas pesquisas do tipo participante ${ }^{(4)}$. Porém, ambas pertencem ao tronco das ciências sociais e, portanto, podem ser vistas com mesma origem metodológica, constituindo alternativa ao padrão de pesquisa convencional.

A ssim, pode-se admitir que toda pesquisa-ação é, por excelência, participante, mas o contrário não ocorre, pois a segunda utiliza a obser vação participante como técnica básica do estudo, enquanto que, na pesquisa-ação, o foco principal situa-se na real ização de reuniões e seminários formados por pesquisadores e sujeitos envolvidos.

Para dar maior singularidade a esse tipo de pesquisa, três elementos são mencionados pelos autores frente à definição do termo: a participação dos sujeitos, a democracia no processo e a contribuição dada às ciências sociais e à mudança social. 0 caráter participativo é condição fundamental para a pesquisa-ação; demanda que os sujeitos envolvidos percebam a necessidade de mudança e queiram exercer papel ativo nesta. A democracia usualmente requer que os participantes sejam vistos como iguais ao pesquisador. Como resultado deste tipo de pesquisa tem-se sua capacidade de influenciar positivamente a prática dos sujeitos investigados no decorrer do estudo, que será compartilhada com um público mais amplo, refletindo seu caráter social( ${ }^{(4)}$. 
Compreende-se então que um estudo adotará a metodologia da pesquisa-ação quando houver necessidade de mudanças no contexto social, evidenciadas pelo estudo e discussão do problema observado, conseqüente às reuniões dos atores envolvidos (pesquisadores e participantes), consistindo numa ação não-trivial ${ }^{(4)}$, sendo capaz de transformar o cenário atual.

\section{PLANEJAMENTO E ELABORAÇÃO DA PESQUISA-AÇÃO}

A elaboração de uma pesquisa-ação segue modelo essencialmente flexível. E mbora contenha fases ordenadas, seu planejamento pode darse de maneira sequencial ou simultânea, dependendo da dinâmica relacional do grupo de pesquisadores com a situação problematizadora a ser investigada.

U m modelo para descrever a metodologia da pesquisa-ação consiste no diagrama de representação em quatro fases ${ }^{(8)}$. A través do desenho proposto, pesquisas que visam investigar o campo empírico e agir sobre este formam dois pólos extremos: pólo investigação e pólo ação, que se encaixam nas características do ciclo: planejar - agir descrever - avaliar, denominado "ciclo da investigação-ação".

E $m$ linhas gerais, o diagrama tem em um de seus lados o pólo investigação, no qual se identifica o problema social e suas possíveis soluções, e no lado oposto o pólo ação, onde decorre todo o processo de inter venção. Limitados por esses pólos, 0 ciclo apresenta-se em 4 fases, sendo a fase 1 (planejar) 0 momento em que são estabel ecidas as metas para mudança da realidade; na fase 2 (agir) as ações planejadas são executadas; na fase 3 (descrever) há o acompanhamento do al cance dessas ações e na fase 4 (avaliar) ocorre a aval iação dos resultados atingidos, iniciando-se novo ciclo.

Didaticamente, o ciclo da pesquisa-ação está disposto em três fases, denominadas fases de ação, assim consider adas par a dar um encadeamento mais ordenado e clarificar as fases da investigação-ação anteriormente mencionada ${ }^{(8)}$.

A ssim, o ciclo da pesquisa-ação contempla todas as atividades do ciclo básico de investigaçãoação e utiliza técnicas de pesquisa consag radas para informar a ação que se decide tomar para meIhorar a prática, contendo técnicas que atendam aos critérios exigidos em outros tipos de pesquisa acadêmica, tais como significância, originalidade e validade ${ }^{(8)}$.

Sob outro prisma, outro estudo da temática também estabelece fases no planejamento da pesquisa-ação, porém reconhece que as mesmas também são flexíveis, não seguindo obrigatoriamente uma ordenação rígida (4). Assim, existem diversos caminhos para sua organização e execução, considerando também a existência de pólos extremos, denominados ponto de partida e ponto de chega$\mathrm{da}^{(4,6)}$, que devem ser rigorosamente preservados para validar o método.

0 ponto de partida ou primeira fase da pesquisa-ação, proposta no estudo citado anteriormente (fase exploratória), representa a descoberta do campo empírico, no qual se situam os sujeitos da investigação e os problemas existentes. Esse é 0 momento dedicado ao estabel ecimento dos primeiros contatos com esses sujeitos, dos quais os pesquisadores passam a identificar expectativas e problemas específicos, estabelecendo um diálogo situacional, enquanto os demais membros da equipe de pesquisa coletam informações relacionadas à temática do estudo (literatura e documentação pertinentes). N essa fase, estabelecem-se os objetivos da pesquisa, considerando-se os problemas prioritários, o campo empírico, os participantes e as ações planejadas(4).

A segunda fase consiste em concretizar o tema da pesquisa e sua del imitação. D ivergente dos métodos convencionais, na pesquisa-ação esta etapa realiza-se a partir de um processo de discussão promovidos entre pesquisadores e participantes. T em-se, portanto, a formulação do tema e sua delimitação, que se apresenta com característica descritiva, pelo fato de pormenorizar a realidade fenomênica, bem como normativa ${ }^{(4)}$, pois a mel horia que se busca pela transformação da real idade sempre supõe um ideal de realidade elaborado pelos sujeitos do estudo.

A pós a definição do tema del imitado, o próximo passo (fase 3: colocação dos problemas) consiste em especificar os problemas para os quais a investigação será direcionada. E stes problemas são, no caso da pesquisa-ação, a priori, de ordem prática. Significa que a pesquisa irá buscar soluções para transfor mar uma situação observada(4).

Outro ponto fundamental da pesquisa-ação (fase 4: o lugar da teoria) consiste em adotar um referencial teórico que dê sustentação às discussões e interpretações emergentes das reuniões e 
seminários realizados e fundamente o raciocínio do grupo. Pesquisadores e participantes devem utilizar a referência teórica em todas as fases da pesquisa por ser útil na formulação dos argumentos que devem ser considerados no percurso investigativo, além de conferir cientificidade ao método ${ }^{(3,6)}$.

T ambém o uso de hipóteses (fase 5) se faz pertinente nesta modal idade de pesquisa. Todavia, a formulação das hipóteses diverge daquela elaborada nas pesquisas convencionais, pelo fato de não pretender estabelecer relação causal entre variáveis. A hipótese qualitativa na pesquisa-ação pretende estabelecer que uma variável tenha relação com outra, porém, com implicações não-causais, cuja verificação se dá unicamente no contexto social alvo da ação(4).

E m seguida (fase 6), inicia-se a realização de seminários, compostos por pesquisadores e sujeitos da investigação, onde os assuntos debatidos em cada sessão devem ser registrados em atas e anal isados posteriormente. Sob esse aspecto, o campo de observação a ser investigado e transformado deve ser específico (fase 7: campo de observação, amostragem e representatividade qualitativa), principal mentese seu tamanho for muito grande ${ }^{(4)}$.

$\mathrm{N}$ ão há consenso sobre a necessidade de utilização dos critérios de amostragem e representatividade na pesquisa-ação, pois al guns autores consideram que o resultado satisfatório da mudança coletiva só será al cançado se contemplar a totalidade da população envolvida; porém, outros estudiosos são favoráveis ao uso de técnicas estatísticas de seleção dos participantes que representarão o conjunto da população. Há ainda uma terceira posição em que se sugere o uso de critérios qualitativos de escol ha dos participantes, consistindo na seleção intencional daqueles considerados informantes-chave, que exercem papel especial no campo empírico(4).

D efinidos os atores (sujeitos participantes), esses passarão a se reunir com os pesquisadores para discutirem os problemas vislumbrados e tomarem decisões. D urante essas reuniões (seminários), a coleta de dados (fase 8) é efetuada, através da utilização de procedimentos e instrumentos diversos, tais como a entrevista coletiva, entrevista individual e anotações em diário de campo, observação participante, histórias de vida, sociodrama e outras técnicas consideradas antropológicas, além dos instrumentos adotados nos estudos convencionais, como o questionário ${ }^{(4)}$. N essa proposta, étam- bém usuais o emprego de técnicas documentais, como jornais, filmes e outros materiais audiovisuais relacionados.

Fruto das discussões e tomada de decisão fundamentada pelo referencial teórico visitado, a aprendizagem do grupo (fase 9) consolida-se, formando um corpus teórico-prático, em que há combinação entre o conhecimento teórico dos pesquisador es com o saber dos participantes. N esse ínterim, todos os envolvidos apropriam-se do saber produzido, havendo a fusão saber formal/ saber informal (fase 10), a fim de combater a prevalência de um sobre 0 outro(4).

D e posse do novo saber e com vistas ao alcance das metas estabelecidas no ponto de partida da investigação, o próximo passo consiste na el aboração de um plano de ação (fase 11), através do qual, pesquisadores e participantes el aboram o que precisa ser feito ou transformado para solucionar 0 problema investigado. D iversos são os tipos de ação que emanam desse tipo de pesquisa: educativa, comunicativa, técnica, política, cultural, de caráter individual ou coletivo(4). N ecessário se faz que seja especificado o tipo de ação proposta, para que todos os envolvidos no processo possam raciocinar em sentido único e possam avaliar posteriormente essas ações.

Como última etapa da pesquisa-ação ou ponto de chegada (fase 12), ocorre à divulgação externa dos resultados da pesquisa aos participantes, bem como em encontros de natur eza científica e nos meios de comunicação acadêmica (revistas, jornais e demais publicações seriadas)(6). Conferese o resultado satisfatório da pesquisa a partir da transformação da realidade ou da conscientização por parte dos envolvidos no estudo. Especificamente nas pesquisas de ação comunicativa, na qual se intenciona elaborar um material próprio de comunicação (panfleto, cartilha, jor nal, vídeo) sua criação ou funcionamento significam o resultado positivo da investigação(4).

\section{A PESQUISA-AÇÃO NA PRÁTICA INVEST IG AT IVA DO ENFERMEIRO}

I nicialmente aplicada nas áreas de educação, comunicação social, serviço social, organização e tecnologia, a pesquisa-ação encontra-se agregando valor a partir de sua utilização numa variedade de disciplinas baseadas na prática(5). A nalise-se 0 fato de que sua utilização tem sido crescente nas 
pesquisas em saúde, especialmente no tocante ao processo de cuidado humano, podendo ser visualizada na medicina preventiva, medicina do trabaIho e no campo da saúde mental|(4), bem como nos diversos espaços do exercício profissional do enfermeiro, seja na saúde col etiva, assistência hospitalar ou docência ${ }^{(6)}$. Isto contribui para que a metodologia al ternativa seja um método bastante eficaz e apropriado para a produção do conhecimento científico do enfermeiro, além de propiciar a combinação de conhecimentos técnicos e sociais entre pesquisadores e participantes.

Especificamente no âmbito da Enfermagem, diversas são as questões de pesquisa que podem ser investigadas, esclarecidas e/ ou resolvidas por intermédio da pesquisa-ação. 0 envolvimento dos profissionais nesse processo garante não apenas maiores possibilidades de solucionar os problemas rotineiros encontrados na prática profissional, mas também a obtenção de diferentes conhecimentos que tenham maior significância para os sujeitos envolvidos no processo(5).

E mbora considerada uma metodologia em uso crescente é ainda escassa nas publicações de Enfermagem e entre os estudos publicados. Todavia, é possível observar que a pesquisa-ação tem sido adotada em algumas situações que envolvem a assistência direta ao cl iente, nas atividades de educação em saúde e na prática docente, denotando a abrangência do método na prática investigativa do enfermeiro.

A assistência de enfermagem pode ser investigada por intermédio da modalidade denominada investigação convergente-assistencial, através da qual o enfermeiro, ao estabelecer a relação terapêutica na prestação do cuidado, identifica problemas de saúde, lista prioridades para solucioná-los e avalia 0 al cance de suas ações na transformação da situação do cliente sob seus cuidados. E sse tipo de modalidade, pela sua característica primordial, que é a coleta de dados freqüente realizada pelo enfermeiro para conduzir sua prática assistencial, constitui um tipo de pesquisa-ação(3), aplicável em qualquer situação que exija cuidado de Enfermagem, uma vez que tem estreita relação com o processo assistencial, viabilizado pel o processo deE $n$ fermagem.

$\mathrm{N}$ a esfer a do gerenciamento, a pesquisa-ação teve alcance positivo em estudo realizado por um grupo de professoras do Departamento de Orientação Profissional da Escola de Enfermagem da
U niver sidade de São Paulo (U SP), que buscou analisar o processo e reestruturar 0 instrumento de avaliação de desempenho profissional de Enfermagem do Hospital Universitário (HU) da referida U niversidade ${ }^{(11)}$.

N o campo da educação em saúde, outro estudo desenvolvido com um grupo de gestantes que freqüentavam o ambulatório do mesmo hospital univer sitário, teve o objetivo de verificar a percepção das integrantes sobre a metodologia da pesquisa-ação, sendo considerada estratégia positiva pelas entrevistadas ${ }^{(12)}$.

Como atividade comunicativa, a pesquisa-ação real izada por enfermeiros e docentes de cursos de enfer magem pode concretizar-se com a el abor ação de material didático ou outras formas de comunicação (jornais, panfletos, cartilhas, vídeos), com a finalidade de desencadear transformações não somente dos participantes, mas de outros g rupos, essencialmente sob o aspecto discursivo.

O utro campo de atuação dessa modal idade de pesquisa é no ensino superior. As escolas de enfermagem encontram, pelo uso da pesquisa-ação, soluções relativas ao processo ensino-aprendizagem, não somente na relação entre professor e al uno, mas também entre professores das diversas disciplinas.

E m estudo desenvolvido por professoras do curso de Enfermagem da U niversidade Estadual de Londrina, Paraná, ministrantes da disciplina $F$ undamentos de Enfermagem, per mitiu verificar que, embora utilizando a denominação de pesquisa participante, essa metodologia permitiu um melhor planejamento das atividades didáticas da disciplina, a partir de reuniões semanais nas quais os problemas identificados eram postos em discussão entre os membros, e as propostas para solucioná-los acatadas por todos ${ }^{(13)}$.

T ambém tem sido aplicada para criar espaço para empowerment de mulheres, como foi o estudo desenvolvido com um grupo de mulheres brasileiras imigrantes em M elbourne, Austrália. N este estudo, por meio da pesquisa-ação criou-se um pessoal e político capaz de acionar o empow erment das participantes, através da filiação em grupo, dos laços comunitários e da ação coletiva ${ }^{(14)}$.

Evidencia-se, portanto, a adequação da pesquisa-ação nas investigações em E nfer magem, independente do modelo estrutural adotado, em virtude da sua aplicabilidade ver sátil e abrangente no cotidiano de trabalho do enfermeiro. Seja no delineamento assistencial executado em ambientes de 
prestação do cuidado, em atividades educativas ou de gerenciamento de ser viços, a pesquisa-ação tem sido utilizada pelo enfermeiro-pesquisador e os resultados positivos emergem desse processo em escal a crescente, fato que vem despontando nas publicações da categoria.

N ecessário se faz que, ao optar pela metodologia alternativa, que o enfermeiro execute suas etapas com o rigor do método(6), validando seus achados fenomênicos, transformando o contex to social estudado e corroborando assim para fortalecer 0 reconhecimento social da Enfermagem e socializando o conhecimento produzido, pois "produzir e divulgar o conhecimento com o objetivo de apresentar à comunidade científica a construção do saber e fazer, em enfermagem, é hoje uma necessidade, tendo em vista a importância, no cenário mundial, dos méritos da profissão que são destacados cada vez mais por meio das publicações dos enfermeiros" ${ }^{\prime \prime}(15)$

\section{CONSIDERAÇÕES FINAIS}

A proposta central do presenteartigo foi propor uma reflexão sobre a pesquisa-ação como um tipo de metodologia de pesquisa empregada por estudiosos de diversos campos do saber e que vem sendo adotada progressivamente por pesquisadores enfermeiros quando da necessidade de realizar o processo investigativo na práxis de enfermagem. Essa metodologia, por ser uma estratégia de pesquisa composta por ciclo ou fases, permite ao enfermeiro adotá-la nos estudos que possuam maior período de tempo para serem desenvolvidos. Por se tratarem de passos flexíveis, suas etapas podem ser alteradas à medida que a realidade empírica modifica seu contexto.

Considera-se que o enfer meiro tem decisivo papel a desempenhar no processo de produção do conhecimento científico de sua profissão, a partir do exercício investigativo que pode, e deve abranger metodologias alternativas para melhor instrumentalizar o raciocínio crítico desse profissional nos campos de atuação, quer seja na assistência de enfermagem, no processo de educação em saúde ou no exercício da docência.

Assim, a metodologia da pesquisa-ação proposta nessa reflexão pode contribuir para potencializar resultados de pesquisas em enfermagem nas quais os sujeitos envolvidos fazem parte do contexto coletivo dos problemas evidenciados, e são capazes, com a utilização desse método, de explorar, agir, aval iar e consequentemente transformar o contexto do grupo de participantes, contribuindo para a conscientização coletiva no processo de mudança.

\section{REFERENCIAS}

1 Angerami M CS. 0 mister da investigação do enfermeiro. Rev Latino-A m Enfermagem. 1993;1(1):11-2.

2 M inayo M CS, organizadora. Pesquisa social: teoria, método e criatividade. Petrópolis: Vozes; 2002.

3 L eopardi M T. M etodol ogia da pesquisa em saúde. F lorianópolis: U niversidade Federal de Santa Catarina; 2002.

4 T hiollent M . M etodologia da pesquisa-ação. São PauIo: Cortez; 2008.

$5 \mathrm{M}$ eyer J. Pesquisa qualitativa na atenção à saúde. Porto Alegre: Artmed; 2005.

6 G rittem L, M eier M J, Zagonel IPS. Pesquisa-ação: uma alter nativa metodológica para pesquisas em enfermagem. Texto Contexto Enferm. 2008;17(4):765-70.

7 Engel GI. Pesquisa-ação. E ducar. 2000;16:181-91.

8 T ripp D. Pesquisa-ação: uma introdução metodológica. E duc Pesqui. 2005;3(31):443-66.

9 M onceau G. T ransformar as práticas para conhecêlas: pesquisa-ação e profissionalização docente. E duc Pesqui. 2005;3(31):467-82.

10 M iranda M G, Resende ACA. Sobre a pesquisa-ação na educação e as armadilhas do praticismo. Rev Bras E duc. 2006;33(11):511-8.

11 G onçal ves VL M , Leite M M J, Ciampone M H T . A pesquisa-ação como método para reconstrução de um processo de avaliação de desempenho. Cogitare Enferm. 2004;1(9):50-9.

12 H oga LAK, R eberte LM . Pesquisa-ação como estratégia para desenvolver grupo de gestantes: a percepção dos participantes. Rev Esc Enferm U SP. 2007; 41(4):559-66.

13 G uariente M HD M , Barbel NA N . A pesquisa participante na formação didático-pedagógica de professores de enfermagem. Rev Latino-Am E nfermagem. 2000;8(2):53-9. 
14 Silva A L . Pesquisa-ação participante no processo de empowerment de mulheres brasileiras no contexto da migração internacional. Esc Anna N ery Rev Enferm [ Internet]. 2008 [ citado 2010 mar 14]; 12(4):750-7. Disponível em: http:// www.revenf.bvs. $\mathrm{br} / \mathrm{pdf} / \mathrm{ean} / \mathrm{v} 12 \mathrm{n} 4 / \mathrm{v} 12 \mathrm{n} 4 \mathrm{a} 20 . \mathrm{pdf}$.

15 Pedro E NR. Divulgando o conhecimento em enfermagem [ editorial] . Rev G aúcha E nferm [ Internet] 2006 [ citado 2010 mar 14] ;27(2):145. D isponível em: http:/ / www.revenf.bvs.br/ pdf/ rge/ v27n2/ v27n2a 01.pdf.

\section{AGRADECIMENT OS}

Ao Conselho $\mathrm{Nacional}$ de Desenvolvimento $\mathrm{Ci}$ entifico e Tecnológico (CN Pq), pelo financiamento do Projeto "Intervenções Ter apêuticas em Saúde M ental: uma proposta para redução do estigma" (processo $n^{\circ}$ 5752012008-8), do qual se originou o presente estudo.
Endereço da autora / Dirección del autor /

\section{Author's address:}

$M$ aria Rosilene Cândido $M$ oreira

Rua Cicero E duardo, s/ n, Bairro J unco

64600-000, Picos, PI

E-mail: rosilene@ ufpi.edu.br
Recebido em: 06/ 04/ 2009

A provado em: 21/ 12/ 2009 